\title{
A decomposition procedure based on approximate Newton directions
}

\begin{abstract}
The efficient solution of large-scale linear and nonlinear optimization problems may require exploiting any special structure in them in an efficient manner. We describe and analyze some cases in which this special structure can be used with very little cost to obtain search directions from decomposed subproblems. We also study how to correct these directions using (decomposable) preconditioned conjugate gradient methods to ensure local convergence in all cases. The choice of appropriate preconditioners results in a natural manner from the structure in the problem. Finally, we conduct computational experiments to compare the resulting procedures with direct methods.
\end{abstract}

\section{Introduction}

The optimization literature has been interested in studying and developing procedures that solve optimization problems with some special structure, by taking advantage of this structure to improve their performance. Examples of special structures that may be exploited in the original model are network constraints, integer and continuous variables, dynamic dependencies, etc. Even in those cases where no special structure is present, it may be imposed externally to use distributed computation resources for the solution of very large problems. The growth in the size and complexity of optimization models during the past years, and the increasing availability of hardware and software for distributed computation, has led to an expansion of this interest.

The traditional approach to take advantage of these characteristics of the problems is based on the application of decomposition techniques. Many different decomposition techniques have been proposed during the past forty years. Early proposals were based on linear programming and theoretical results from convex analysis, such as the well-known Dantzig-Wolfe decomposition [10] and its dual variant, Benders decomposition [3]. Another frequently used technique is the Lagrangian Relaxation procedure $[15,21,24]$, based on convex analysis theory and requiring the use of nondifferentiable optimization procedures to maximize the dual function. Relaxation techniques based on

A.J. Conejo: E.T.S. de Ingenieros Industriales, Univ. de Castilla-La Mancha, Ciudad Real, Spain, e-mail: aconejo@ind-cr.uclm.es

F.J. Nogales: E.T.S. de Ingenieros Industriales, Univ. de Castilla-La Mancha, Ciudad Real, Spain, e-mail: fjnogale@ind-cr.uclm.es

F.J. Prieto: Dept. of Statistics and Econometrics, Univ. Carlos III de Madrid, Spain, e-mail: f jpeest-econ . uc3m.es 
Augmented Lagrangian functions [9,4] allow the use of differentiable techniques and extensions to nonconvex cases by combining penalization methods with local duality theory, at the expense of additional problem manipulations to ensure separability [28, $22,23]$. Finally, other decomposition techniques take the structure of the problem into account to specialize classical optimization procedures, such as $[5,8,7]$. These techniques achieve a high level of efficiency, at the price of being strongly dependent on the particular form of the problem.

In some cases, these procedures based on relaxation techniques may present drawbacks: difficulties to converge to an optimal solution (in the absence of convexity assumptions), and convergence rates that are very sensitive to the choice of values for the parameters [24, 18]. Moreover, these techniques may require the computation of a very precise solution of the subproblems resulting from each set of values of the parameters in each iteration. As a consequence, the computational effort may be quite high, particularly for large-scale problems.

Our interest in this paper is to derive a general procedure for the solution of large-scale nonlinear and possibly nonconvex problems, that is able to take advantage of special structures that may be present in the problem. The recent development of interior-point techniques for the solution of linear and quadratic problems has implied that these problems can be efficiently solved by transforming them into nonlinear (although generally convex) problems. In this sense, the methods that we will describe should be useful for the solution of general (linear and nonlinear) continuous optimization problems with special structure.

Our proposal will not follow the traditional decomposition approaches described above. Instead, it will be based on modifications of the procedure to compute the Newton directions, that is, the directions of improvement obtained from the solution of the corresponding KKT systems of linear equations. The proposal will depend crucially on the observation that the special structures of interest will also be apparent both in the derivatives of the functions and in the coefficient matrices of the KKT systems.

The method we propose is thus a procedure to obtain a modified Newton direction that is computed from decomposed systems of linear equations. It is based on exploiting the problem structure present in the KKT systems in an efficient manner to approximate them by separable linear systems. In this sense, it is related to the many proposals in the literature for the distributed solution of linear systems of equations, see for example [26]. The proposed technique is also adapted to the fact that we wish to solve a sequence of related linear systems, one for each iterate. We do not need a very precise solution for each one of these systems, as a sufficient approximation to Newton's direction is enough to ensure reasonable convergence properties in the algorithm. In this regard, the proposed procedure is also related to optimization methods based on the inexact solution of the subproblems, see for example [11].

The resulting decomposition methodology is simple, having very few parameters to consider, efficient and very well suited for its implementation in a distributed computation environment. On the other hand, the approximations introduced to decompose the problem imply that the superlinear rate of convergence of a pure Newton approach will in general become a linear rate of convergence for the decomposition algorithm. This reduction in the convergence rate is often in practice more than offset by the computational gains achieved through the solution of the smaller problems obtained from the 
decomposition. The linear rate of convergence can be improved using a preconditioned conjugate gradient (PGC) method $[17,16]$ for the solution of the linear systems of equations. In particular, we will use a generalized minimal residual (GMRES) procedure [27, 17], as the coefficient matrix of the system of equations will not be positive definite in general.

Although this approach may seem to be unrelated to traditional decomposition methods, we will see that it is close to Lagrangian decomposition techniques in that it solves decomposed subproblems obtained after fixing some of the primal and dual variables in the Lagrangian function. Nevertheless, the updating rules for these fixed variables differ from those standard in Lagrangian decomposition methods.

The method will be particularly useful whenever the special structure of the problem fits one of the two following patterns, that will be used as our references in the remainder of the paper:

- Complicating constraints. Problems with complicating constraints arise frequently in numerous applications, including multicommodity network flows (in scheduling and transportation models), allocation of scarce resources among competing activities [1], design and management of water supply systems and electric power network analysis [30], logistics, econometric data fitting and statistics [2], etc. These models can be written in the general form

$$
\begin{array}{cl}
\operatorname{minimize} & f\left(x_{1}, \ldots, x_{N}\right) \\
\text { subject to } & h_{0}\left(x_{1}, \ldots, x_{N}\right) \leq 0 \\
& c_{j}\left(x_{j}\right) \leq 0 \quad j=1, \ldots, N,
\end{array}
$$

where the constraints (2) are referred to as complicating constraints; if they were removed, the resulting problem would be separable.

- Complicating variables. Problems with complicating variables appear in scenario analysis and stochastic optimization [25], financial planning under uncertainty [20] and structural optimization, among others. The corresponding models can be written as

$$
\begin{array}{cl}
\operatorname{minimize} & f\left(x_{0}, x_{1}, \ldots, x_{N}\right) \\
\text { subject to } & h_{j}\left(x_{0}, x_{j}\right) \leq 0 \quad j=1, \ldots, N \\
& c_{0}\left(x_{0}\right) \leq 0
\end{array}
$$

where the variables $x_{0}$ in (4)-(6) are referred to as complicating variables; if they were fixed to constant values, the resulting problem would be separable.

In both cases $x_{j} \in \mathbb{R}^{n_{j}}$, and $f$ and each $h_{j}, c_{j}$ are smooth functions for $j=$ $0, \ldots, N$, with $f: \mathbb{R}^{n} \rightarrow \mathbb{R}, h_{j}: \mathbb{R}^{n} \rightarrow \mathbb{R}^{m_{h_{j}}}$ and $c_{j}: \mathbb{R}^{n_{j}} \rightarrow \mathbb{R}^{m_{c_{j}}}$, where $n=\sum_{j} n_{j}$

The remainder of this paper is organized as follows: In Section 2 we will motivate the proposed procedure as a modified Lagrangian relaxation technique. Section 3 describes the proposed procedure and studies its local convergence properties. In Section 4 we present different preconditioner choices for the proposed algorithm. We also describe 
in Section 5 practical implementation details and computational results from the application of this procedure to the solution of both nonlinear and linear problems. Finally, Section 6 states some conclusions.

\section{A modified Lagrangian relaxation procedure}

In this section, we try to motivate the proposed procedure by considering a particular implementation of Lagrangian relaxation for the complicating constraints case, (1)-(3). In Section 3 we will show that this implementation is a particular case of the more general decomposition procedure we describe in this paper.

To simplify our discussion, we consider the case in which we have only two groups of variables $(N=2)$ and additionally all constraints are equality ones. The simplified problem will have the form

$$
\begin{array}{cl}
\operatorname{minimize} & f\left(x_{1}, x_{2}\right) \\
\text { subject to } & h_{1}\left(x_{1}, x_{2}\right)=0 \\
& h_{2}\left(x_{1}, x_{2}\right)=0 \\
& c_{j}\left(x_{j}\right)=0 \quad j=1,2,
\end{array}
$$

where we have introduced some partition of the constraints $h_{0}$ into $h_{1}$ and $h_{2}$.

The basic Lagrangian procedure applied to this problem considers the auxiliary problem

$$
\begin{array}{cl}
\operatorname{minimize} & f\left(x_{1}, x_{2}\right)-\bar{\lambda}_{1}^{T} h_{1}\left(x_{1}, x_{2}\right)-\bar{\lambda}_{2}^{T} h_{2}\left(x_{1}, x_{2}\right) \\
\text { subject to } & c_{j}\left(x_{j}\right)=0 \quad j=1,2,
\end{array}
$$

defined in terms of multiplier estimates $\bar{\lambda}_{1}$ and $\bar{\lambda}_{2}$. Problem (11)-(12) can be solved by fixing the values of some of the variables $\left(\bar{x}_{2}\right.$ and $\left.\bar{x}_{1}\right)$ to obtain the subproblems

$$
\begin{array}{ll}
\text { minimize } & f\left(x_{1}, \bar{x}_{2}\right)-\bar{\lambda}_{1}^{T} h_{1}\left(x_{1}, \bar{x}_{2}\right) \\
\text { subject to } & c_{1}\left(x_{1}\right)=0,
\end{array}
$$

and

$$
\begin{array}{cl}
\operatorname{minimize} & f\left(\bar{x}_{1}, x_{2}\right)-\bar{\lambda}_{2}^{T} h_{2}\left(\bar{x}_{1}, x_{2}\right) \\
\text { subject to } & c_{2}\left(x_{2}\right)=0 .
\end{array}
$$

Once the solutions for these subproblems have been computed, the multipliers of the complicating constraints can be updated, using for example a subgradient technique,

$$
\left(\bar{\lambda}_{1}\right)_{k+1}=\left(\bar{\lambda}_{1}\right)_{k}-\alpha h_{1}\left(x_{1}, x_{2}\right), \quad\left(\bar{\lambda}_{2}\right)_{k+1}=\left(\bar{\lambda}_{2}\right)_{k}-\alpha h_{2}\left(x_{1}, x_{2}\right) .
$$

Note that the convergence of the procedure requires that the solutions for the subproblems should be computed up to a certain degree of accuracy.

The procedure proposed in this paper follows a similar approach when applied to problem (7)-(10). As in the preceding case, to decompose problem (11)-(12) we require 
some separable approximation for both $f, h_{1}$ and $h_{2}$. We also fix some of the variables in these functions to their last computed values, to obtain

$$
\begin{array}{cl}
\operatorname{minimize} & f\left(x_{1}, \bar{x}_{2}\right)-\bar{\lambda}_{2}^{T} h_{2}\left(x_{1}, \bar{x}_{2}\right) \\
\text { subject to } & h_{1}\left(x_{1}, \bar{x}_{2}\right)=0 \\
& c_{1}\left(x_{1}\right)=0,
\end{array}
$$

and

$$
\begin{array}{cl}
\operatorname{minimize} & f\left(\bar{x}_{1}, x_{2}\right)-\bar{\lambda}_{1}^{T} h_{1}\left(\bar{x}_{1}, x_{2}\right) \\
\text { subject to } & h_{2}\left(\bar{x}_{1}, x_{2}\right)=0 \\
& c_{2}\left(x_{2}\right)=0,
\end{array}
$$

where $\bar{x}_{1}$ and $\bar{x}_{2}$ denote the values of the corresponding variables at the last iterate. To reduce the computational cost, instead of solving the subproblems to optimality we perform a single Newton step (we compute one search direction and perform one linesearch) for each subproblem. The values of the variables resulting from this step are then used to update the parameters $\bar{x}_{1}$ and $\bar{x}_{2}$.

This procedure is not very different from a standard Lagrangian approach, except for performing a single iteration for each subproblem, but it presents one significant advantage: it provides efficient information to update the multiplier estimates $\bar{\lambda}_{1}$ and $\bar{\lambda}_{2}$. The multipliers corresponding to the subproblem constraints (16) and (17), $\Delta \lambda_{1}$ and $\Delta \lambda_{2}$, have the property that, if the values of $\bar{x}_{1}$ and $\bar{x}_{2}$ would be the optimal ones, the best values for $\lambda_{1}$ and $\lambda_{2}$ would be given by $\bar{\lambda}_{1}+\Delta \lambda_{1}$ and $\bar{\lambda}_{2}+\Delta \lambda_{2}$. These updated values can be used for the next iteration.

The resulting procedure is very simple to implement, uses few easily updated parameters and does work well in practice for certain classes of problems (see Section 5.2). As a consequence, it seems reasonable to study conditions under which this simplified Lagrangian decomposition scheme would converge. In the following sections we will present a decomposition scheme that generalizes the procedure described above to solve different classes of problems, and we analyze its convergence properties.

\section{Proposed algorithm}

The algorithm we briefly described in the preceding section uses specific procedures to update the values of the variables and multipliers from one iteration to the next. In this section we wish to expand this procedure into a more general decomposition algorithm that is efficient to implement and requires as few parameters as possible, while preserving the basic schemes introduced in the preceding section, that is, to approximate the functions in the problem by fixing some of the variables to attain separability.

To simplify our presentation, the description of this algorithm will be based on the complicating constraints model (1)-(3). At the end of the section we will indicate how to extend the corresponding results to the complicating variables model (4)-(6).

We will assume that problem (1)-(3) will be solved using interior-point techniques. These procedures simplify the description of the algorithm by transforming the problem 
into an equality constrained one; they are also computationally very efficient, particularly for large-scale problems. Thus, the optimization problem will be solved through a sequence of barrier problems in which the inequality constraints have been rewritten as equalities using slack variables, and the bounds on the variables have been removed via the addition of barrier terms to the objective function. For a description of the use of these techniques in the general nonconvex case see for example [31, 12, 32, 13].

The resulting model, to be used as our reference in the description of the decomposition procedure, will have the general form

$$
\begin{array}{cl}
\operatorname{minimize} & f\left(x_{1}, \ldots, x_{N} ; \mu\right) \\
\text { subject to } & h\left(x_{1}, \ldots, x_{N}\right)=0 \\
& c_{j}\left(x_{j}\right)=0 \quad j=1, \ldots, N,
\end{array}
$$

where we assume $x_{j} \in \mathbb{R}^{n_{j}}, f: \mathbb{R}^{n} \rightarrow \mathbb{R}, h: \mathbb{R}^{n} \rightarrow \mathbb{R}^{m_{h}}, c_{j}: \mathbb{R}^{n_{j}} \rightarrow \mathbb{R}^{m_{c_{j}}}$, $n=\sum_{j} n_{j}$. The function $f$ includes the barrier terms corresponding to the bounds on the variables, and $\mu$ denotes the corresponding barrier parameter. These terms are not relevant for the description of the decomposition procedure, as in the barrier terms we take into account only simple bounds on the variables (all other constraints are transformed into equalities), and the resulting terms are trivially separable.

An implicit assumption for decomposition procedures is that the number of complicating constraints is not very large, that is, $m_{h} \ll \sum_{j} m_{c_{j}}$. The description of the algorithm does not require that this condition holds, but the efficiency of our procedure will depend on these values, as we will discuss in Section 5.1.

In this paper we are mostly interested in the analysis of the local convergence of the proposed decomposition procedure. As a consequence, the algorithm introduced in this section will not consider mechanisms to ensure global convergence, such as line searches or trust regions. The scheme of this basic algorithm is presented in Figure 1, where the values $\sigma_{j}$ and $\lambda$ will denote the multiplier estimates for the constraints $c_{j}\left(x_{j}\right)=0$ and $h(x)=0$, respectively. We will denote by $x$ the vector of all (primal) variables, $x=\left(x_{1}^{T}, \ldots, x_{N}^{T}\right)^{T}$, while $\sigma$ denotes the vector $\sigma=\left(\sigma_{1}^{T}, \ldots, \sigma_{N}^{T}\right)^{T}$. In Figure 1 , the positive constant $\epsilon$ represents a termination tolerance and the function $L$ denotes the Lagrangian function of problem (18)-(20).

No explicit procedure is given for the update of the barrier parameter $\mu$. We will assume that any of the procedures proposed in the literature, see [31, 12] for example, is used to update this parameter. In this sense, we will be mostly concerned with the (local) convergence of the procedure for a fixed value of $\mu$.

The decomposition procedure depends crucially on the definition of the inner iteration, that is, the procedure to compute the search direction $\Delta_{k}$. Our method of reference for this inner iteration is Newton's method; in this setting, in each outer iteration $k$ the search direction is computed by solving the following system of linear equations:

$$
\left(\begin{array}{ccc}
\nabla^{2} L(x, \sigma, \lambda) & \nabla c^{T}(x) & \nabla h^{T}(x) \\
\nabla c(x) & 0 & 0 \\
\nabla h(x) & 0 & 0
\end{array}\right)\left(\begin{array}{c}
\Delta x \\
-\Delta \sigma \\
-\Delta \lambda
\end{array}\right)=-\left(\begin{array}{c}
\nabla L(x, \sigma, \lambda) \\
c(x) \\
h(x)
\end{array}\right),
$$




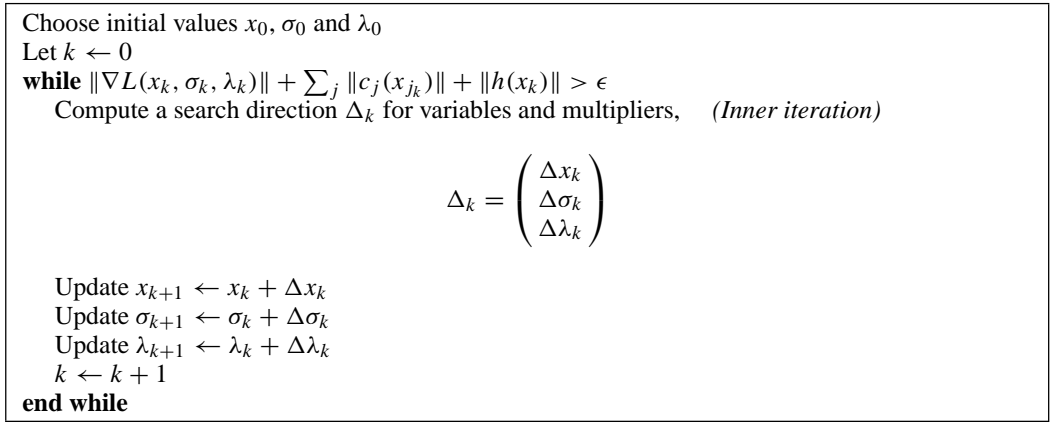

Fig. 1. Basic algorithm

where

$$
\nabla c(x)=\left(\begin{array}{lll}
\nabla c_{1}(x) & & \\
& \ddots & \\
& & \nabla c_{N}(x)
\end{array}\right),
$$

and all quantities are evaluated at the current iterate (the subscript $k$ corresponding to the iteration has been omitted to simplify the notation).

Observe that, within an interior point framework, this representation would correspond to a primal approach. Primal-dual approaches are considered to be more efficient than primal ones, but for a primal-dual approach the resulting linear system would be equivalent to (21), after simplifying the update for the dual variables. Both systems would only differ in the diagonal terms for the first block of coefficients. Again, this does not affect the decomposition procedure and we will ignore these details in the description of the inner iteration.

In Section 2 we introduced an approach for solving problems of this kind, based on Lagrangian relaxation. That procedure proceeded by fixing the values of some of the variables to obtain decomposable subproblems, and then used the solutions of these subproblems to update the fixed variables. This approach is equivalent to replacing the Newton system of linear equations for problem (18)-(20) by a separable system that approximates it. In particular, fixing variables in the proposed Lagrangian relaxation approach is equivalent to approximating the matrix $\nabla^{2} L$ in (21) by a block diagonal matrix, while the matrix $\nabla h$ is replaced by a matrix having separable blocks.

To generalize this approach, note that the special structure in the original problem appears also in the constraints, in the sense that the linear system would be separable if two conditions were met: i) the constraints $h(x)=0$ would be separable (would have a separable Jacobian matrix) or would not exist; and ii) the matrix $\nabla^{2} L$ would be separable, that is, it would be block diagonal with blocks corresponding to the different groups of variables; this would be the case if both $f$ and $h$ are separable. In general, these conditions are not satisfied, although in many practical cases the departures from them are not large. The proposed method replaces (21) with a related and separable system, of the form 


$$
\left(\begin{array}{ccc}
H & \nabla c^{T}(x) & A^{T} \\
\nabla c(x) & 0 & 0 \\
A & 0 & 0
\end{array}\right)\left(\begin{array}{c}
\Delta x \\
-\Delta \sigma \\
-\Delta \lambda
\end{array}\right)=-\left(\begin{array}{c}
\nabla L(x, \sigma, \lambda) \\
c(x) \\
h(x)
\end{array}\right),
$$

where $H$ and $A$ are approximations to the Hessian matrix $\nabla^{2} L$ and the Jacobian $\nabla h$, respectively, having the property of being separable in the variables $x_{j}$. We will use the abbreviated notation $K \Delta_{N}=-g$ for system (21), and $\bar{K} \Delta_{m}=-g$ for the modified system (23).

The matrices $H$ and $A$ can be obtained by setting to zero a sufficient number of elements in the preceding matrices to ensure separability. For example, the terms in $\nabla^{2} L$ corresponding to cross derivatives for variables belonging to different blocks could be replaced by zeros, while the constraints in $\nabla h$ could be divided into groups associated with each block of variables; the nonzero coefficients in each set of constraints not associated with the corresponding block of variables could then be set to zero. This is very similar to the approach used in Section 2 , where the zeros were introduced by fixing the values of some of the variables in the subproblems.

We now analyze sufficient conditions (on the search directions) to guarantee the local convergence of the method shown in Figure 1.

\subsection{A simple inner iteration}

If system (21) is solved exactly to compute $\Delta_{N}$, we have the standard Newton algorithm, that has assured local convergence under certain assumptions on the problem. As system (21) is not separable, we will not use this approach. Alternatively, consider a simplified inner iteration where at iteration $k$ we define

$$
\Delta_{k} \equiv \Delta_{m, k}=-\bar{K}_{k}^{-1} g_{k} .
$$

In some cases $\Delta_{m}$ may be close enough to $\Delta_{N}$ to preserve some of the local convergence properties of Newton's method. This will happen if $\bar{K}_{k}$ is sufficiently close in some sense to $K_{k}$, that is, if the modification required in the system to make it separable is small. In these cases the problem can be directly decomposed by ignoring some of the elements in $\nabla^{2} L$ and $\nabla h$ in the computation of the search directions, without taking any additional correction steps and without losing local convergence. We now give a result based on classical results from the theory of iterative methods for linear systems of equations, see for example [29], that provides sufficient conditions to ensure local convergence in this simplified setting.

For the remainder of this section, we will assume that problem (18)-(20) satisfies the following hypotheses. For a given second-order KKT point of problem (18)-(20), $y^{*}=\left(x^{*}, \sigma^{*}, \lambda^{*}\right)$, it holds that:

A.1 The functions $f, c_{j}, h$ have Lipschitz-continuous second derivatives in an open set containing $y^{*}$.

A.2 The Jacobian of the constraints, $\left(\nabla c_{1}^{T}\left(x_{1}\right) \ldots \nabla c_{N}^{T}\left(x_{N}\right) \nabla h^{T}(x)\right)^{T}$, has full row rank at $y^{*}$.

A.3 The sufficient second-order optimality conditions for problem (18)-(20) are satisfied at $y^{*}$.

We will also require the approximations $\bar{K}_{k}$ to satisfy the following condition: 
$C .1$ The matrices $\bar{K}_{k}$ are nonsingular for any $y_{k}$, and the sequence $\left\{\bar{K}_{k}\right\}_{k}$ converges to a nonsingular matrix $\bar{K}^{*}$ as $y_{k} \rightarrow y^{*}$.

This condition is imposed to ensure that the solution of the system (24) should be well defined at all iterations, and its behavior should be reasonable near the solution. Any factorization approach that controls the ill-conditioning in the system should ensure its satisfaction.

In the following theorem $K^{*}$ denotes the matrix $K$ evaluated at $y^{*}$, and for any given matrix $A, \rho(A)$ denotes its spectral radius.

Theorem 1. Under assumption A.1 and condition C.1, if at the second-order KKT point $y^{*}$ it holds that

$$
\rho^{*}=\rho\left(I-\left(\bar{K}^{*}\right)^{-1} K^{*}\right)<1,
$$

then the procedure using (24) converges locally to $y^{*}$ with linear rate at least equal to $\rho^{*}$.

Proof. Let $y_{k}$ be an iterate that is sufficiently close to $y^{*}$ so that assumption A.1 holds and $K_{k}$ is bounded. From the corresponding Taylor series expansion,

$$
g_{k}=g^{*}+K^{*}\left(y_{k}-y^{*}\right)+o\left(\left\|y_{k}-y^{*}\right\|\right),
$$

where $g^{*}=0$. Also, by definition in the algorithm $y_{k+1}=y_{k}+\Delta_{k}$ and $\Delta_{k}=-\bar{K}_{k}^{-1} g_{k}$. Consequently,

$$
y_{k+1}-y^{*}=y_{k}+\Delta_{k}-y^{*}=\left(I-\bar{K}_{k}^{-1} K^{*}\right)\left(y_{k}-y^{*}\right)+o\left(\left\|y_{k}-y^{*}\right\|\right)
$$

and taking norms

$$
\left\|y_{k+1}-y^{*}\right\| \leq\left\|I-\bar{K}_{k}^{-1} K^{*}\right\|\left\|y_{k}-y^{*}\right\|+o\left(\left\|y_{k}-y^{*}\right\|\right) .
$$

This result together with the condition on the spectral radius implies that the sequence $\left\{y_{k}-y^{*}\right\}$ converges to zero. Dividing by $\left\|y_{k}-y^{*}\right\|$ and taking limits, the result on the rate of convergence follows.

A consequence of this result is that certain problems, even if they are not directly separable, can be solved efficiently by computing a search direction from a separable system of equations (the one defined by $\bar{K}$ ). In particular, the proposed method based on the Lagrangian relaxation technique that we described in Section 2 would fit this framework and would be locally convergent under the conditions of Theorem 1 .

Note that the convergence criterion in Theorem $1, \rho^{*}<1$, is the classical condition for the convergence of iterative methods for the solution of systems of linear equations, such as iterative refinement, Jacobi or Gauss-Seidel. It is not easy in general to check in advance if this condition will hold for a given problem. But the convergence condition of the decomposition algorithm and that for the convergence of the iterative refinement method are the same, and this fact would provide a simple way to check the convergence condition at a given iteration as the algorithm progresses. If iterative refinement generates convergent iterates for the KKT system at a sequence of iterations of the decomposition procedure, then the full algorithm should also converge. 


\subsection{A locally convergent algorithm}

Our next step will be to develop a decomposition algorithm that is locally convergent, independently of the satisfaction of condition (25) on the approximating matrix $\bar{K}_{k}$. To attain this goal we will use a modification of the simple inner iteration proposed above, based on adding correction steps to the direction $\Delta_{m, k}$.

To compute $\Delta_{m, k}$ we need some factorization of the matrix $\bar{K}_{k}$, and this is a computationally expensive procedure. It would seem reasonable to obtain these correction steps through procedures that do not require any additional factorizations. A simple way to do this is to apply an appropriate version of the preconditioned conjugate gradient (PCG) method (see [17]) to system (21), as the solution of this system has the desired local convergence properties. A natural choice for the preconditioner is the matrix $\bar{K}_{k}$, whose factors would be already available. As the matrix $K$ is not positive definite (except perhaps in the unconstrained convex case), we have chosen to use GMRES (see [27]), a variant of the PCG procedure that does not impose any condition on the coefficient matrix of the system.

In each (outer) iteration $k$, the movement direction $\Delta_{k}$ is defined from the inner iteration indicated in Figure 2. In this inner iteration $t_{k}$ denotes a positive termination tolerance to be specified later on; for the convergence analysis we only require $0<t_{k} \leq \bar{t}<1$ for all $k$ and some constant $\bar{t}$. Note that, within the inner iteration, all systems of equations have $\bar{K}$ as their coefficient matrix; in consequence they can be solved in a distributed manner.

The termination condition for the inner iteration is given in terms of the residuals of the system of Newton equations. This criterion has been chosen as it is easy to implement, it enforces the local convergence of the overall procedure, as we will see below, and it is efficient in practice. We now study the local convergence of the modified method. Note first that, as GMRES solves the system of equations $K_{k} \Delta_{k}=-g_{k}$, from the convergence of GMRES and condition $C .1$, the termination condition for the inner iteration is satisfied in a finite number of (inner) iterations.

We now establish that, close to a second-order KKT point $y^{*}$ for problem (18)-(20), the method is locally convergent. As a first step, we start by relating the sizes of $g_{k}$ and $\Delta_{k}$.

Lemma 1. Under assumptions A.1 to A.3, if $y_{k}$ is close enough to $y^{*}$, then there exist constants $r_{1}$ and $r_{2}$ such that

$$
\left\|g_{k}\right\| \leq r_{1}\left\|\Delta_{k}\right\|, \quad\left\|g_{k}\right\| \geq r_{2}\left\|\Delta_{k}\right\| .
$$

$$
\begin{aligned}
& \text { Solve } \bar{K}_{k} \Delta_{m, k}=-g_{k} \\
& \text { Let } \tilde{\Delta}_{0} \leftarrow \Delta_{m, k} \text { and } i \leftarrow 0 \\
& \text { while }\left\|K_{k} \tilde{\Delta}_{i}+g_{k}\right\|>t_{k}\left\|g_{k}\right\| \\
& \quad \text { Apply one iteration of GMRES using } \bar{K}_{k} \text { as preconditioner } \\
& \quad \text { to compute } \tilde{\Delta}_{i+1} \\
& \quad i \leftarrow i+1 \\
& \text { end while } \\
& \Delta_{k} \leftarrow \tilde{\Delta}_{i}
\end{aligned}
$$

Fig. 2. Inner iteration 
Also, there exists a constant $r_{3}$ such that

$$
\left\|\Delta_{k}\right\| \leq r_{3}\left\|y_{k}-y^{*}\right\|
$$

Proof. From the termination condition we must have

$$
\left\|g_{k}+K_{k} \Delta_{k}\right\| \leq t_{k}\left\|g_{k}\right\|
$$

Also, from assumption $A .1$ it will hold that $\left\|K_{k}\right\| \leq \bar{r}$ for some constant $\bar{r}$ and all $y_{k}$ close enough to $y^{*}$. Consequently, we will have that

$$
t_{k}\left\|g_{k}\right\| \geq\left\|g_{k}\right\|-\bar{r}\left\|\Delta_{k}\right\| \Rightarrow\left\|g_{k}\right\| \leq \frac{\bar{r}}{1-\bar{t}}\left\|\Delta_{k}\right\| .
$$

From assumptions $A .1$ to $A .3$ it must follow that, for all $y_{k}$ close enough to $y^{*}$, the smallest singular value of $K_{k}$ is bounded away from zero. Let $\tilde{r}>0$ be such a bound. Then

$$
\left\|g_{k}+K_{k} \Delta_{k}\right\| \geq\left\|K_{k} \Delta_{k}\right\|-\left\|g_{k}\right\| \geq \tilde{r}\left\|\Delta_{k}\right\|-\left\|g_{k}\right\|,
$$

and from (26),

$$
(1+\bar{t})\left\|g_{k}\right\| \geq \tilde{r}\left\|\Delta_{k}\right\|
$$

implying the second inequality.

Finally, from this second inequality and

$$
g_{k}=g^{*}+O\left(\left\|y_{k}-y^{*}\right\|\right)=O\left(\left\|y_{k}-y^{*}\right\|\right),
$$

we obtain the third inequality.

We are now ready to prove a local convergence result for this algorithm.

Theorem 2. Under assumptions A.1 to A.3 and condition C.1, the sequence $\left\{y_{k}\right\}$ generated using the algorithm in Figure 2 converges locally to $y^{*}$ with linear rate of convergence no larger than $\bar{t}$.

Proof. If $y_{k}$ is close enough to $y^{*}$, from Lemma 1 and assumption $A .1$

$$
g_{k+1}=g_{k}+K_{k}\left(y_{k+1}-y_{k}\right)+o\left(\left\|y_{k+1}-y_{k}\right\|\right)=g_{k}+K_{k} \Delta_{k}+o\left(\left\|\Delta_{k}\right\|\right) .
$$

Taking norms and using the termination condition for the inner iteration (26) and Lemma 1 ,

$$
\left\|g_{k+1}\right\| \leq\left\|g_{k}+K_{k} \Delta_{k}\right\|+o\left(\left\|g_{k}\right\|\right) \leq t_{k}\left\|g_{k}\right\|+o\left(\left\|g_{k}\right\|\right) .
$$

From this inequality it follows that $g_{k} \rightarrow 0$, and from Lemma 1 this implies $y_{k} \rightarrow y^{*}$. Dividing by $\left\|g_{k}\right\|$ and taking limits it follows from Lemma 1 that the rate of convergence is at least equal to $\bar{t}$.

From this result, the choice of $t_{k}$ should offer a compromise between the rate of convergence of the algorithm, and consequently the number of outer iterations required for convergence, and the computational cost of each outer iteration. In section 5.1 additional information will be provided on how to choose $t_{k}$ from a practical point of view. 


\subsection{The complicating variables case}

The preceding paragraphs have analyzed problems with complicating constraints. We consider now the complicating variables case (4)-(6) in a similar manner. If we apply a barrier approach to the solution of this problem, we obtain the resulting model

$$
\begin{array}{cl}
\operatorname{minimize} & f\left(x_{0}, x_{1}, \ldots, x_{N} ; \mu\right) \\
\text { subject to } & h_{j}\left(x_{0}, x_{j}\right)=0 \quad j=1, \ldots, N \\
& c_{0}\left(x_{0}\right)=0,
\end{array}
$$

where the objective function includes the relevant barrier terms. If we apply Newton's method, the corresponding KKT system of equations (at a given iteration) would have the form

$$
\left(\begin{array}{ccc}
\nabla^{2} L(x, \sigma, \lambda) & \nabla h^{T}(x) & \nabla c_{0}^{T}\left(x_{0}\right) \\
\nabla h(x) & 0 & 0 \\
\nabla c_{0}\left(x_{0}\right) & 0 & 0
\end{array}\right)\left(\begin{array}{c}
\Delta x \\
-\Delta \sigma \\
-\Delta \lambda
\end{array}\right)=-\left(\begin{array}{c}
\nabla L(x, \sigma, \lambda) \\
h(x) \\
c_{0}\left(x_{0}\right)
\end{array}\right),
$$

where now $x=\left(x_{0}^{T} x_{1}^{T} \cdots x_{N}^{T}\right)^{T}, \sigma_{j}$ denotes the multiplier for $h_{j}\left(x_{0}, x_{j}\right), \sigma=$ $\left(\sigma_{1}^{T} \cdots \sigma_{N}^{T}\right)^{T}, \lambda$ is the multiplier for $c_{0}\left(x_{0}\right)=0$ and

$$
\nabla h(x)=\left(\begin{array}{cccc}
\nabla_{x_{0}} h_{1} & \nabla_{x_{1}} h_{1} & & \\
\vdots & & \ddots & \\
\nabla_{x_{0}} h_{N} & & & \nabla_{x_{N}} h_{N}(x)
\end{array}\right) .
$$

The procedures described for problem (18)-(20), and in particular the construction of the approximate system (23), can be applied in the same manner to approximate this KKT system, to obtain

$$
\left(\begin{array}{ccc}
H & A^{T} \nabla c_{0}^{T}\left(x_{0}\right) \\
A & 0 & 0 \\
\nabla c_{0}\left(x_{0}\right) & 0 & 0
\end{array}\right)\left(\begin{array}{c}
\Delta x \\
-\Delta \sigma \\
-\Delta \lambda
\end{array}\right)=-\left(\begin{array}{c}
\nabla L(x, \sigma, \lambda) \\
h(x) \\
c_{0}\left(x_{0}\right)
\end{array}\right),
$$

for some approximations $H$ and $A$ to $\nabla^{2} L$ and $\nabla h$ respectively. These approximations should be separable in the variables $x_{0}, x_{1}, \ldots, x_{N}$. For example, the blocks that correspond to the variables $x_{0}$ in $\nabla h$ could be made equal to zero (if that would not imply a loss of rank), as well as the blocks in $\nabla^{2} L$ associated with cross derivatives for $x_{0}$ and $x_{i}$ with $i \neq 0$. The algorithms described in Figures 1 and 2 could be applied with only the obvious modifications to adapt them to (30) and (31).

\section{Preconditioning}

The efficiency of the procedures described in Section 3 depends crucially on an appropriate choice of the preconditioner $\bar{K}_{k}$ for the KKT system in each iteration of the 
proposed method. More specifically, the convergence of the procedure in Figure 2 will depend on the proximity of $\bar{K}_{k}^{-1} K_{k}$ to the identity matrix $I$. If we write

$$
\bar{K}_{k}^{-1} K_{k}=I+B_{k},
$$

then we have two criteria to select a good preconditioner in terms of the properties of the matrix $B_{k}$ in (32).

- The matrix $B_{k}$ has a small spectral radius. From Theorem 1 convergence would follow if $\sigma_{\max }\left(B_{k}\right)<1$, where $\sigma_{\max }\left(B_{k}\right)$ denotes the largest singular value of $B_{k}$. The rate of local convergence would depend on the magnitude of this value, that is, it would be faster as $\sigma_{\max }\left(B_{k}\right)$ becomes smaller.

- The matrix $B_{k}$ is a perturbation matrix of small rank. If exact arithmetic is used, the number of inner (GMRES) iterations required to find the exact solution of the system of linear equations (the Newton direction) is at most $r=\operatorname{rank}\left(B_{k}\right)+1$, see [16] for example; more precisely, it is at most equal to the number of different singular values of $B_{k}$. In practice, if these singular values are clustered in a small number of groups (for example, if many of them are equal to zero) then the preconditioned GMRES procedure would behave efficiently. This is the case for example if only a few of the rows and columns of $K_{k}$ are modified to obtain $\bar{K}_{k}$.

Note also that the preconditioner must satisfy condition $C .1$, and in particular it must be a full-rank matrix.

Based on these remarks, we now present alternative preconditioner choices for the algorithm in Figure 2. These choices are based on replacing some of the entries in the KKT matrix, $K_{k}$, by zeros. If these entries are chosen appropriately, the resulting system can be trivially decomposed.

1. The complicating constraints can be decomposed by introducing zeros in $\nabla h$ to obtain a separable matrix. For example, by partitioning $h$ into two subsets of constraints $h_{1}$ and $h_{2}$, we could define the matrix $A$ in (23) as (again assuming $N=2$ for simplicity)

$$
A=\left(\begin{array}{cc}
\nabla_{x_{1}} h_{1} & 0 \\
0 & \nabla_{x_{2}} h_{2}
\end{array}\right) .
$$

If this can be done without reducing the rank of $A$ below that of $\nabla h$, then the matrix $\bar{K}_{k}^{-1} K_{k}$ has at most $2 m_{h}$ eigenvalues different from one, and the rest of eigenvalues are equal to 1 . The rank of matrix $B_{k}$ in (32) is $2 m_{h}$.

We have found that this procedure works reasonably well for problems with complicating constraints, but it usually leads to reduced-rank preconditioner matrices in problems with complicating variables.

2. An alternative approach that complements the preceding one if there are difficulties with the rank of the preconditioner, proceeds by replicating the complicating constraints (19) $N$ times, if the resulting number of equations would not exceed the number of variables. The KKT matrix, $K_{k}$, would have the form (we assume $N=2$ for simplicity)

$$
\left(\begin{array}{cc}
\nabla^{2} \tilde{L} & \nabla \tilde{h}^{T} \\
\nabla \tilde{h} & 0
\end{array}\right)
$$


where

$$
\nabla^{2} \tilde{L}=\left(\begin{array}{cc}
\nabla^{2} L & \nabla c^{T}(x) \\
\nabla c(x) & 0
\end{array}\right)
$$

and

$$
\nabla \tilde{h}=\left(\begin{array}{ccc}
\nabla_{x_{1}} h & \nabla_{x_{2}} h & 0 \\
\nabla_{x_{1}} h & \nabla_{x_{2}} h & 0
\end{array}\right)
$$

The right-hand side would be replicated in a similar manner. The preconditioner, $\bar{K}$, would have the form

$$
\bar{K}=\left(\begin{array}{cc}
\tilde{H} & \tilde{A}^{T} \\
\tilde{A} & 0
\end{array}\right)
$$

where

$$
\tilde{A}=\left(\begin{array}{ccc}
A_{1} & 0 & 0 \\
0 & A_{2} & 0
\end{array}\right),
$$

and $\tilde{H}, A_{1}$ and $A_{2}$ are approximations to the Hessian $\nabla^{2} \tilde{L}$ and the Jacobians $\nabla_{x_{1}} h$ and $\nabla_{x_{2}} h$, respectively. It is important to select these matrices, $A_{1}$ and $A_{2}$, in such a way that the resulting preconditioner matrix $\bar{K}$ has full rank. Observe that the KKT matrix $K_{k}$ is singular, but the system is compatible.

If $\tilde{H}, A_{1}$ and $A_{2}$ can be chosen to coincide exactly with the previous Hessian and Jacobian matrices, the matrix $\bar{K}_{k}^{-1} K_{k}$ would have $2 m_{h}$ eigenvalues equal to zero, $2 m_{h}$ eigenvalues equal to 2 , and the remaining eigenvalues are equal to one. The resulting matrix $B_{k}$ in (32) has rank $4 m_{h}$.

This approach seems to work well in practice in problems with complicating variables, but is less adequate for problems with complicating constraints, as we will comment in Section 5.

The local convergence theory of section 3.2 can be extended to this case, even if assumption $A .2$ is not satisfied after the constraints are replicated. We still need this assumption, as well as assumptions $A .1$ and $A .3$, to hold for the original problem (18)(20). Let $\Delta \lambda_{k}=\Delta \lambda_{k}^{1}+\Delta \lambda_{k}^{2}$, where $\Delta \lambda^{1}$ and $\Delta \lambda^{2}$ denote the multiplier estimates for each set of replicated constraints. The convergence theory in Lemma 1 and Theorem 2 will hold if we consider the sequences $\left\{\Delta x_{k}\right\}$ and $\left\{\Delta \lambda_{k}\right\}$, instead of $\left\{\Delta x_{k}\right\},\left\{\Delta \lambda_{k}^{1}\right\}$ and $\left\{\Delta \lambda_{k}^{2}\right\}$ (the sequences generated by the algorithm based on the replication of constraints), and $K_{k}$ and $g_{k}$ refer to the original system of equations, before the duplication of constraints.

\section{Numerical results}

In this section we describe the implemented versions of the algorithm, and present numerical results obtained by applying the proposed decomposition algorithm to a set of test problems. 


\subsection{Practical implementation}

Several versions of the algorithm (using different preconditioners $\bar{K}$ ) have been implemented in Matlab to test its behavior on linear and nonlinear problems with special structure. The implementation is based on the description in Figures 1 and 2, but it includes a few additional details that are included in Figure 3.

The following issues are of particular interest:

- The proposed algorithm carries out several iterative refinement iterations, while the norm of the residuals is decreasing. In practice, this is a cheap way to improve the quality of the computed direction, and it works quite well in many problems for which it holds that $\rho\left(\bar{K}^{-1} K\right)<1$.

- The initial value for the termination tolerance for GMRES, $t_{k}$, is chosen in terms of the behavior of the problem in the first iteration.

- The termination tolerance $t_{k}$ is updated dynamically taking into account the rate of reduction in the residual norm $r_{k}$. This strategy is very similar to that used in trust-region methods to adjust the size of the region and works well in practice.

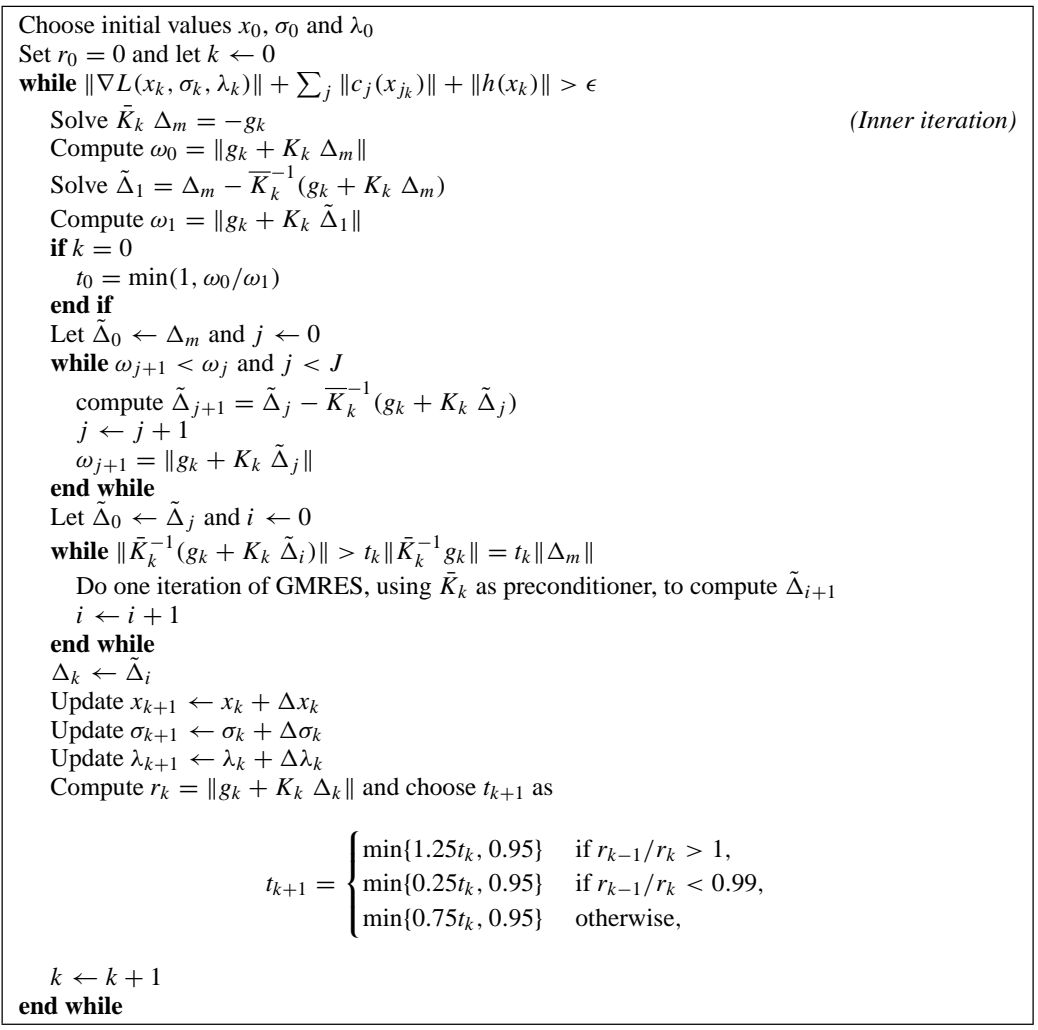

Fig. 3. Decomposition algorithm 


\subsection{Test problems and results}

We have tested the algorithm on a set of test problems from two models, one linear and one nonlinear, that present both coupling patterns described in Section 1.

The first set of test problems, having complicating variables, is based on a twostage stochastic programming model [6]. This model is a large-scale linear program that minimizes the cost of first-period decisions plus the expected cost of second-period recourse decisions, while satisfying some first-period constraints. The stochastic problem is solved as a deterministic one by considering a discrete distribution with associated probabilities. Under these approximations, the deterministic equivalent form of the problem is

$$
\begin{array}{ll}
\underset{x, y_{1}, \ldots, y_{N}}{\operatorname{minimize}} & c^{T} x+\sum_{i=1}^{N} \pi_{i}\left(q_{i}^{T} y_{i}\right) \\
\text { subject to } & A x=b \\
& B_{k} x+W_{i} y_{i}=h_{i}, \text { for } i=1,2, \ldots, N \\
& x \geq 0, y_{i} \geq 0, \text { for } i=1,2, \ldots, N,
\end{array}
$$

where $x$ denotes the first-period variables, $y_{i}$ denotes variables corresponding to second-period decisions and (34), (35) are the first-period and second-period constraints, respectively.

We have also studied a second set of test problems, corresponding to a complicating constraints case with nonlinear functions. It is based on a multi-area optimal power flow problem (OPF), whose formulation (for the single-area case) is discussed in [30]. The resulting problem is a large-scale non-convex optimization problem. A model for this problem in compact form has the structure given in (1)-(3), where $x_{j}$ would be the variables for each area $j$ of the global system. Equations (2) represent the power flow equations and transmission capacity limits for those buses and lines interconnecting different areas. Constraints (3) include the power flow equations and transmission capacity limits, only for those lines and buses lying within a given area, and limits over dependent and control variables. The sets of equations (2)-(3) represent both equality and inequality constraints. In these models, the objective function (1) is the total operation cost for the system, a quadratic function of $x_{j}$ for all $j$.

Table 1 shows the most relevant characteristics of the problems in the test sets corresponding to each one of the two models. The first column gives the problem name; the second column indicates the number $N$ of areas/scenarios, that will correspond to the number of subproblems in the solution algorithm; a third column shows the number of complicating constraints/variables $c$; the fourth and fifth columns present the total number of variables $n$ and functional constraints $m$, respectively.

The first fifteen problems (the SP problems) correspond to a subset of POSTS, a portable stochastic programming test set [19]. Detailed descriptions for these problems and the data used to define them can be obtained from

http: //www-personal.umich.edu/ jrbirge/dholmes/spProblems Intro.html

The last ten cases (the MOPF problems) are multi-area OPF models. They correspond to standard IEEE bus systems and their description can be found in [14]. 
Table 1. Main characteristics of test problems

\begin{tabular}{|l|rrrr|}
\hline \multicolumn{1}{|c|}{ Case } & \multicolumn{1}{c}{$\mathrm{N}$} & $\mathrm{c}$ & \multicolumn{1}{c|}{$\mathrm{n}$} & \multicolumn{1}{c|}{$\mathrm{m}$} \\
\hline SP1 & 6 & 188 & 1820 & 686 \\
SP2 & 6 & 151 & 2845 & 1520 \\
SP3 & 16 & 188 & 4540 & 1726 \\
SP4 & 16 & 151 & 7335 & 3900 \\
SP5 & 6 & 306 & 8568 & 3353 \\
SP6 & 9 & 306 & 12699 & 4937 \\
SP7 & 32 & 151 & 14519 & 7708 \\
SP8 & 15 & 306 & 20961 & 8105 \\
SP9 & 64 & 151 & 28887 & 15324 \\
SP10 & 30 & 306 & 41616 & 16025 \\
SP11 & 96 & 151 & 43255 & 22940 \\
SP12 & 128 & 151 & 57623 & 30556 \\
SP13 & 45 & 306 & 62271 & 23945 \\
SP14 & 160 & 151 & 71991 & 38172 \\
SP15 & 60 & 306 & 82926 & 31865 \\
\hline MOPF1 & 3 & 39 & 72 & 101 \\
MOPF2 & 2 & 15 & 224 & 167 \\
MOPF3 & 2 & 46 & 128 & 191 \\
MOPF4 & 3 & 25 & 336 & 251 \\
MOPF5 & 3 & 50 & 1032 & 1344 \\
MOPF6 & 6 & 110 & 2064 & 2972 \\
MOPF7 & 6 & 112 & 4428 & 6573 \\
MOPF8 & 12 & 238 & 8856 & 14649 \\
MOPF9 & 24 & 490 & 17712 & 35310 \\
MOPF10 & 50 & 1032 & 36900 & 99212 \\
\hline
\end{tabular}

A primal-dual interior point approach has been used to generate equality constrained problems, both in the form indicated in (18)-(20), for the MOPF problems, and in an equivalent form for the complicating variables SP problems. These problems have been solved using both a direct approach (Newton method) and the decomposition procedure described in figure 3. Both procedures have been initialized using the same starting point, and the same termination tolerances have been applied. The algorithms stopped whenever $\|\nabla L(x, \lambda)\| /(1+\|x\|+\|\lambda\|) \leq 10^{-6}$, where $L$ denotes the Lagrangian function. The choice of preconditioner for the decomposition procedure has been made to ensure that the resulting preconditioner satisfies condition C.1 (the preconditioner has full rank). For problems with complicating constraints, the preconditioner described in item 2 of Section 4 tends to produce matrices $\bar{K}_{k}$ that are rank deficient; as a consequence, for the MOPF problems we have used the preconditioner described in item 1 of Section 4. For problems with complicating variables, the situation is the opposite one, that is, preconditioner 1 tends to yield matrices $\bar{K}_{k}$ that are rank deficient; for the SP problems we have used the preconditioner described in item 2 of Section 4. The sparse LU factorizations provided by Matlab were used in the codes: for the SP problems (problems with complicating variables), an incomplete LU factorization with 0 level of fill-in was implemented, while for the MOPF problems (problems with complicating constraints) the code was based on the LU factorization with column minimum degree permutation. Table 2 presents a comparison of the computational results obtained from both algorithms on the SP problems, while table 3 presents the corresponding results for the MOPF problems.

The columns of tables 2 and 3 correspond to the following information: 
Table 2. Numerical results for the SP problems

\begin{tabular}{|l|lrrcc|}
\hline Case & $I_{g}$ & $I_{d}$ & $I_{C G}$ & $T_{g}$ & $T_{d}$ \\
\hline SP1 & 27 & 27 & 145 & $9.51410^{0}$ & $7.86110^{0}$ \\
SP2 & 20 & 20 & 43 & $3.83510^{1}$ & $2.07510^{1}$ \\
SP3 & 28 & 28 & 291 & $5.80110^{1}$ & $3.70410^{1}$ \\
SP4 & 21 & 22 & 58 & $2.68710^{2}$ & $5.93010^{1}$ \\
SP5 & 30 & 31 & 49 & $2.77810^{2}$ & $1.03310^{2}$ \\
SP6 & 33 & 33 & 55 & $7.04310^{2}$ & $1.83710^{2}$ \\
SP7 & 25 & 25 & 65 & $5.85110^{2}$ & $1.40610^{2}$ \\
SP8 & 38 & 39 & 57 & $2.40710^{3}$ & $4.41610^{2}$ \\
SP9 & 24 & 26 & 71 & $4.18210^{3}$ & $7.04810^{2}$ \\
SP10 & 43 & 43 & 93 & $1.17810^{4}$ & $1.96510^{3}$ \\
SP11 & 29 & 31 & 73 & $1.75010^{4}$ & $2.01810^{3}$ \\
SP12 & 29 & 29 & 84 & $5.17410^{4}$ & $2.51410^{3}$ \\
SP13 & 41 & 42 & 97 & $3.94110^{4}$ & $2.45510^{3}$ \\
SP14 & 32 & 33 & 112 & $9.12710^{4}$ & $4.58310^{3}$ \\
SP15 & 39 & 39 & 98 & $1.25210^{5}$ & $7.11810^{3}$ \\
\hline
\end{tabular}

Table 3. Numerical results for the MOPF problems

\begin{tabular}{|l|rrrll|}
\hline \multicolumn{1}{|c|}{ Case } & $I_{g}$ & $I_{d}$ & $I_{C G}$ & \multicolumn{1}{c|}{$T_{g}$} & \multicolumn{1}{c|}{$T_{d}$} \\
\hline MOPF1 & 22 & 23 & 74 & $2.80510^{-1}$ & $2.79810^{-1}$ \\
MOPF2 & 21 & 21 & 10 & $3.12910^{-1}$ & $2.83510^{-1}$ \\
MOPF3 & 23 & 42 & 391 & $3.51910^{-1}$ & $1.34110^{0}$ \\
MOPF4 & 26 & 26 & 57 & $6.11910^{-1}$ & $6.66110^{-1}$ \\
MOPF5 & 36 & 35 & 15 & $7.37110^{0}$ & $6.31510^{0}$ \\
MOPF6 & 43 & 39 & 9 & $3.10810^{1}$ & $2.18910^{1}$ \\
MOPF7 & 37 & 39 & 27 & $8.68310^{1}$ & $8.77110^{1}$ \\
MOPF8 & 40 & 41 & 22 & $4.63610^{2}$ & $4.58210^{2}$ \\
MOPF9 & 43 & 43 & 12 & $2.75810^{3}$ & $2.62610^{3}$ \\
MOPF10 & 44 & 43 & 10 & $2.14510^{4}$ & $1.97310^{4}$ \\
\hline
\end{tabular}

- The second and third columns indicate the total number of Newton iterations required by the direct method, $I_{g}$, and the decomposition procedure $I_{d}$, respectively, for each one of the problems. This value corresponds to the number of times systems (21) or (23) must be solved in the algorithm.

- The fourth column gives the total number of conjugate-gradient iterations, $I_{C G}$, performed by the GMRES subroutine. This is also the total number of solves performed by the decomposed algorithm using the preconditioner matrix $\bar{K}$.

- The fifth and sixth columns show the total accumulated CPU time in seconds needed to solve the problems using both the direct approach, $T_{g}$, and the decomposition procedure, $T_{d}$, on a single processor. This time includes the linear algebra time and the time required by the updating of variables and derivative computations in Newton's method. The reported times correspond to a Pentium III at $800 \mathrm{MHz}$ with $1 \mathrm{~GB}$ of memory.

The results in Table 2 show a clear advantage of the proposed decomposition approach over the direct method, even when a single processor is used. It is remarkable to note that, although the proposed procedure has only linear convergence, the total number of iterations hardly increases when the decomposition scheme is applied. All 


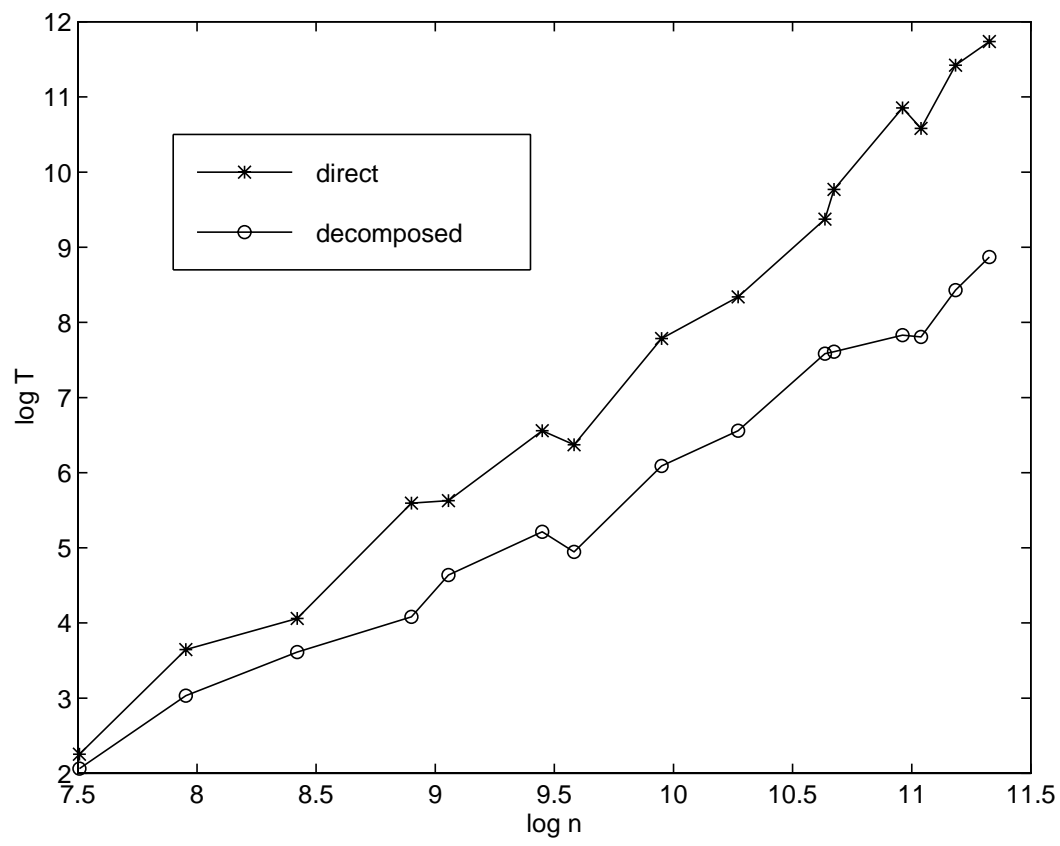

Fig. 4. Logarithms of total running times for both the direct approach $T_{g}$ and the proposed algorithm $T_{d}$, vs. the logarithm of the number of variables $n$ in the problems of the SP test set

CPU times are lower for the proposed procedure, and this improvement increases with the size of the problem. These running times have been plotted in figure 4 , for both the direct approach and the proposed algorithm, against the number of variables in each problem, to emphasize the increase in the improvement with the size of the problem; note the logarithmic scale in the figure.

Table 3 presents the corresponding results for the MOPF problems. These problems are much more demanding for the algorithm, particularly in a sequential setting, as they are nonlinear and $60 \%$ of the CPU solution time on the average is devoted to the computation of derivatives (this percentage goes up to $80 \%$ for the largest problems). As a consequence, any increase in the number of Newton iterations, as is the case for problem MOPF3 for example, has a significant impact on the solution time, while any decreases due to the computation of the search direction in the proposed approach have a more limited impact than for the SP problems. Nevertheless, it is interesting to note that the solution times decrease for seven problems, versus three increases, all of them in smaller problems. Thus, the proposed procedure might seem quite promising for the solution of large problems of this class in a distributed computation environment.

The proposed algorithm described in figure 3 is based on the simple inner iteration described in section 3.1, corresponding to a special case of the Lagrangian decomposition algorithm. This simple iteration is then expanded with a CG iteration to improve the convergence properties of the algorithm. We were also interested in studying the relevance of this last modification, and its impact on the efficiency of the resulting 
Table 4. Numerical results using a simple inner iteration. Problems MOPF

\begin{tabular}{|l|cc|}
\hline \multicolumn{1}{|c|}{ Case } & $I_{d}$ & $T_{d}$ \\
\hline MOPF1 & 59 & $3.69910^{-1}$ \\
MOPF2 & 38 & $4.59010^{-1}$ \\
MOPF3 & & \\
MOPF4 & 58 & $1.00310^{0}$ \\
MOPF5 & 39 & $5.31410^{0}$ \\
MOPF6 & 52 & $2.36610^{1}$ \\
MOPF7 & 49 & $9.62010^{1}$ \\
MOPF8 & 52 & $4.47710^{2}$ \\
MOPF9 & 51 & $2.48310^{3}$ \\
MOPF10 & 48 & $1.41610^{4}$ \\
\hline
\end{tabular}

algorithm. We have conducted a final experiment, reported in table 4, for the set of MOPF problems, solving these problems by applying the algorithm described in section 3.1.

The second and third columns of table 4 indicate the total number of iterations $I_{d}$ required by the decomposition procedure and the CPU time in seconds $T_{d}$ needed to solve the problems, respectively. The blank entry corresponds to a case in which the algorithm did not converge, as the corresponding problem did not satisfy condition (25). It is remarkable to note that so many of the MOPF problems did in fact satisfy this condition, and the simple algorithm was able to obtain solutions for them. Comparing these results with the CPU times indicated in table 3, the running times are slightly higher for the smaller problems, due to the larger number of Newton iterations required, but they are lower for the three larger problems (including a 34\% improvement on the direct solution for the largest one). For the problems in the SP test set the convergence condition was not satisfied, and no comparable results are available. These results may suggest the interest of detecting and exploiting property (25) for those large problems where it may hold.

\section{Conclusions}

We have discussed an algorithm based on an approximate Newton direction, computed from an approximation to the KKT system of equations and a preconditioned conjugate gradient procedure. This algorithm can also be motivated as a particular case of a Lagrangian relaxation procedure. The natural choice of preconditioner based on a decomposable approximation to the system works very well in practice, better than the direct solution of the system in many cases when this is feasible.

Another important issue we have considered is the termination criterion for the inexact computation of the search directions. We propose using a dynamic update of the termination tolerance based on the quality of the preceding directions. This approach results in a small number of conjugate gradient iterations, and also in a reduced number of Newton steps. The resulting procedure seems to have clear advantages over the direct approach whenever the linear algebra cost is the dominant one in the optimization algorithm, for example in the case of linear programs with special structure. It is also promising for the distributed computation of both linear and nonlinear problems with structure. 


\section{References}

1. M. S. Bazaraa, J. J. Jarvis, and H. F. Sherali. Linear Programming and Network Flows. John Wiley and Sons, New York, second edition, 1990.

2. M. S. Bazaraa, H. D. Sherali, and C. M. Shetty. Nonlinear Programming: Theory and Algorithms, John Wiley, New York, second edition, 1993.

3. J. F. Benders. Partitioning procedures for solving mixed variables programming problems. Numer. Math., 4:238-252, 1962.

4. D. P. Bertsekas and J. N. Tsitsiklis. Parallel and Distributed Computation: Numerical Methods. PrenticeHall Inc., 1989.

5. J. R. Birge and D. F. Holmes. Efficient solution of two-stage stochastic linear programs using interior point methods. Computational Optimization and Applications, 1:245-276, 1992.

6. John R. Birge and François Louveaux. Introduction to Stochastic Programming. Springer-Verlag, New York, 1997.

7. J. Castro. A specialized interior-point algorithm for multicommodity network flows, 1998. Acepted for publication in SIAM Journal on Optimization.

8. Y. Censor and S. A. Zenios. Parallel Optimization: Theory, Algorithms, and Applications. Numerical Mathematics and Scientific Computation. Oxford University Press, 1997.

9. G. Cohen. Auxiliary Problem Principle and Decomposition of Optimization Problems. Journal of Optimization Theory and Applications, 32(3), Nov 1980.

10. G. B. Dantzig and P. Wolfe. Decomposition principle for linear programming. Operations Research, 8:101-111, 1960.

11. R. S. Dembo and U. Tulowitzki. On the minimization of quadratic functions subject to box constraints. Working Paper Series B 71, School of Organization and Management, Yale University, New Haven, Conn., 1983.

12. A. S. El-Bakry, R. A. Tapia, T. Tsuchiya, and Y. Zhang. On the formulation and theory of Newton interior-point method for nonlinear programming. Journal of Optimization Theory and Applications, 89(3):507-541, Jun 1996.

13. A. L. Forsgren and P. E. Gill. Primal-dual interior methods for nonconvex nonlinear programming. SIAM Journal on Optimization, 8:1132-1152, 1998.

14. L. L. Freris and A. M. Sasson. Investigation of the load flow problem. Proceedings of the IEE, 115:1459$1470,1968$.

15. A. M. Geoffrion. Lagrangean Relaxation for integer programming. Mathematical Programming Study, 2:82-114, 1974.

16. G. H. Golub and C. F. Van Loan. Matrix Computations. The Johns Hopkins University Press, London, third edition, 1996.

17. M. R. Hestenes and E. Steifel. Methods of conjugate gradients for solving linear systems. Journal of Research National Bureau of Standards, 49:409-436, 1954.

18. J. B. Hiriart-Urruty and C. Lemaréchal. Convex Analysis and Minimization Algorithms, volume I and II. Springer Verlag, Berlin, 1996.

19. D. Holmes. A (po)rtable (s)tochastic programming (t)est (s)et (posts). http://www-personal.umich.edu/ jrbirge/dholmes/post.html.

20. P. Kall and S. W. Wallace. Stochastic Programming. John Wiley, Chichester, UK, 1994.

21. J. E. Kelley. The cutting-plane method for solving convex programs. Journal of the Society for Industrial and Applied Mathematics, 8:703-712, 1960.

22. R. de Leone, R. R. Meyer, S. Kontogiorgis, A. Zakarian, and G. Zaker. Coordination in coarse-grained decomposition. SIAM Journal on Optimization, 4(4):777-793, 1994.

23. K. Park and Y. Shin. Iterative bundle-based decomposition for large-scale nonseparable convex optimization. European Journal of Operations Research, 111:598-616, 1998.

24. B. T. Polyak. Introduction to Optimization. Optimization Software Inc., New York, 1987.

25. R. T. Rockafellar and Roger J.-B. Wets. Scenarios and policy aggregation in optimization under uncertainty. Math. Oper. Res., 16(1):119-147, 1991.

26. Y. Saad. Iterative Methods for Sparse Linear Systems. PWS Publishing, New York, 1996.

27. Y. Saad and M. H. Schultz. GMRES: a generalized minimal residual algorithm for solving nonsymmetric linear systems. SIAM Journal on Scientific and Statistic Computations, 7:856-869, 1986.

28. G. L. Schultz and R. R. Meyer. An interior point method for block angular optimization. SIAM Journal on Optimization, 1(4):583-602, 1991.

29. R. S. Varga. Matrix Iterative Analisys. Prentice-Hall, Englewood Cliffs, NJ, 1962.

30. A. J. Wood and B. F. Wollenberg. Power Generation, Operation and Control. John Wiley and Sons, New York, second edition, 1996.

31. S. J. Wright. Primal-Dual Interior-Point Methods. SIAM Publications, SIAM, Philadelphia, PA, USA, 1996.

32. H. Yamashita and H. Yabe. Superlinear and quadratic convergence of some primal-dual interior point methods for constrained optimization. Mathematical Programming, 75:377-397, 1996. 